\title{
Theoretical analysis of ultra-fast phase recovery in semiconductor optical amplifiers
}

\author{
CHEN LiGong, XU TianXiang, ZHENG Xiu, ZHANG ShangJian, GUO XiaoWei, \\ TANG XiongGui \& LIU Yong
}

School of Optoelectronic Information, University of Electronic Science \& Technology of China, Chengdu 610054, China

Received April 29, 2011; accepted November 22, 2011

\begin{abstract}
We present results from theoretical analysis of the phase dynamics in semiconductor optical amplifiers (SOAs). In particular, we focus on an aspect of the ultra-fast phase recovery that currently does not have adequate in-depth theoretical analysis and clear explanation of the physical mechanism. We build up a numerical model to analyze the ultra-fast phase recovery of semiconductor optical amplifiers in details. To investigate the phase response characteristics, we analyze the different contributions to the phase shift, including intra-band effects such as carrier heating, spectral hole burning, and inter-band effects such as carrier depletion. In addition, the impact of the pulses energy on phase shift is also investigated. Based on the analysis of phase response characteristics, we further explain the reason why a delay occurs between gain response and phase response. The analysis results are in good agreement with the reported experimental results. The results presented in this paper are useful for the SOA-based ultra-fast optical signal processing, such as optical switches, optical logic gates, and optical Add/Drop multiplexer.
\end{abstract}

optical signal processing, nonlinear optics, intra-band physical effects, nonlinear phase shift, semiconductor optical amplifier

Citation: Chen L G, Xu T X, Zheng X, et al. Theoretical analysis of ultra-fast phase recovery in semiconductor optical amplifiers. Chin Sci Bull, 2012, 57: 1078-1082, doi: 10.1007/s11434-011-4967-0

Semiconductor optical amplifiers (SOAs) are intensively used in all-optical wavelength conversion, optical logic gates, 3R regeneration, optical Add/Drop multiplexer due to the large nonlinearity in small power consumption [1-6]. One main drawback of SOAs is the long gain recovery time that limits SOAs in high-speed operation. For example, an $8 \times 8$ InP-based monolithic tunable optical router (MOTOR) chip has been constructed, but operating speeds are still limited to $40 \mathrm{Gbit} / \mathrm{s}$ in a single channel [7]. Many schemes were proposed to enhance SOA response speeds, such as increasing active region length, increasing inject current, inducing continuous wave $(\mathrm{CW})$ holding beam, optimizing geometrical structure [8], and employing blue-detuned filtering technology. Among these schemes, the blue-detuned filtering technology has shown the capability for ultra-high

*Corresponding author (email: yongliu@uestc.edu.cn) speed operation, $320 \mathrm{Gbit} / \mathrm{s}$ error-free all-optical wavelength conversion and $640 \mathrm{Gbit} / \mathrm{s}$ all optical demultiplexing were demonstrated experimentally [1,6]. With bit-rates increasing rapidly, phase response is another factor that should be taken into consideration in SOA-based all-optical switches [9].

The phase response of inter-band effects is already studied intensively [10], but the impact of intra-band effects on phase shift is less concerned and the reported results are also different. Giller et al. [11] studied the phase dynamics of bulk SOAs using terahertz optical asymmetric demultiplexer (TOAD) loop; the results showed that the intra-band effects yield few contributions to the phase shift. In [12], the experimental results showed about 2 ps ultra-fast phase recovery under the injection of sub-picosecond pump pulses. The disparate results show that the ultra-fast phase recovery process in the SOAs is related to the operating conditions. 
At present, there lacks an in-depth theoretical investigation and a clear physical mechanism explanation of the ultra-fast phase recovery in the SOAs.

The beam propagation method is effective in the study of field characteristics in SOAs [13]. In this paper, we develop a numerical model based on carrier-rate equation and beam propagation method to analyze the phase characters of the SOAs. In the numerical model, ultra-fast carrier dynamics such as carrier heating $(\mathrm{CH})$, spectral hole burning (SHB), two-photon absorption (TPA) and free-carrier absorption (FCA) are included. Group velocity dispersion and gain dispersion are also taken into count for short input pump pulses.

\section{Theoretical model of SOA}

Considering the various contributions to the SOA gain, such as carrier density change, $\mathrm{CH}$ and $\mathrm{SHB}$, the SOA total gain has three contributing components

$$
g=g_{\mathrm{N}}+\Delta g_{\mathrm{CH}}+\Delta g_{\mathrm{SHB}},
$$

where $g_{\mathrm{N}}, \Delta g_{\mathrm{CH}}$ and $\Delta g_{\mathrm{SHB}}$ are the gain change related to carrier density change, $\mathrm{CH}$ and $\mathrm{SHB}$, respectively. These are given by

$$
\begin{gathered}
g_{\mathrm{N}}=\alpha_{0}\left(n_{\mathrm{c}}^{*}+n_{\mathrm{v}}^{*}-n_{0}\right), \\
\Delta g_{\mathrm{CH}}=\alpha_{0}\left(\overline{n_{\mathrm{c}}}-n_{\mathrm{c}}^{*}+\overline{n_{\mathrm{v}}}-n_{\mathrm{v}}^{*}\right), \\
\Delta g_{\mathrm{SHB}}=\alpha_{0}\left(n_{\mathrm{c}}-\overline{n_{\mathrm{c}}}+n_{\mathrm{v}}-\overline{n_{\mathrm{v}}}\right),
\end{gathered}
$$

where $a_{0}$ is the gain coefficient, $n_{0}$ the total density of states in the optical coupled region, $n_{x}^{*}(x=\mathrm{c}, \mathrm{v}$, for conduction and valence band respective) the local carrier densities of conduction and valence bands under lattice temperature, and $n_{x}(x=\mathrm{c}, \mathrm{v})$ are the local carrier densities of conduction and valence bands. Below, we use $\mathrm{x}$ to index the two types of bands c and v. Densities $n_{\mathrm{c}}$ and $n_{\mathrm{v}}$ evolve according to

$$
\begin{gathered}
\frac{\partial n_{\mathrm{c}}(z, \tau)}{\partial \tau}=\frac{\overline{n_{\mathrm{c}}}-n_{\mathrm{c}}}{\tau_{\mathrm{c}}}-v_{\mathrm{g}} g(z, \tau) S(z, \tau)-n_{\mathrm{c}} \beta_{\mathrm{c}} v_{\mathrm{g}} S(z, \tau), \\
\frac{\partial n_{\mathrm{v}}(z, \tau)}{\partial \tau}=\frac{\overline{n_{\mathrm{v}}}-n_{\mathrm{v}}}{\tau_{\mathrm{v}}}-v_{\mathrm{g}} g(z, \tau) S(z, \tau)-n_{\mathrm{v}} \beta_{\mathrm{v}} v_{\mathrm{g}} S(z, \tau),
\end{gathered}
$$

where $\overline{n_{x}}$ are the quasi-equilibrium values, $\tau_{x}$ are the carrier-carrier scatter time in the conduction and valence bands, $\beta_{x}$ are the free-carrier absorption coefficients, $v_{\mathrm{g}}$ is the group velocity, and $s(z, \tau)$ is the photon number density.

The SOA gain dispersion is taken into account in the numerical model because the injection of the short pump pulses $(<10 \mathrm{ps})$ that causes wide spectral range [14]. Hence, the group velocity dispersion is also introduced in the model because the SOA phase $\varphi$ is related to gain by linewidth enhancement factor $\alpha$ expressible as

$$
\frac{\partial \Phi}{\partial z}=-\frac{1}{2} \alpha g
$$

Assuming the gain spectrum of SOAs is of parabolic type, the SOA gain can be expanded in a Taylor series

$$
g(\tau, \omega)=g\left(\tau, \omega_{0}\right)+\left.\Delta \omega \frac{\partial g(\tau, \omega)}{\partial \omega}\right|_{\omega_{0}}+\left.\frac{\Delta \omega^{2}}{2} \frac{\partial^{2} g(\tau, \omega)}{\partial \omega^{2}}\right|_{\omega_{0}} .
$$

In eq. (8), the first-order differential $\left.\frac{\partial g(\tau, \omega)}{\partial \omega}\right|_{\omega_{0}}$ and the second-order differential: $\left.\frac{\partial^{2} g(\tau, \omega)}{\partial \omega^{2}}\right|_{\omega_{0}}$ can be simplified as [15]

$$
\begin{gathered}
\left.\frac{\partial g(\tau, \omega)}{\partial \omega}\right|_{\omega_{0}}=A_{1}+B_{1}\left[g_{0}-g(\tau, \omega)\right], \\
\left.\frac{\partial^{2} g(\tau, \omega)}{\partial \omega^{2}}\right|_{\omega_{0}}=A_{2}+B_{2}\left[g_{0}-g(\tau, \omega)\right],
\end{gathered}
$$

where $A_{1}$ and $A_{2}$ is the slope of the SOA linear gain at $\omega_{0} ; B_{1}$ and $B_{2}$ is the rate of gain change resulting from saturation effect.

Based on the above analysis results and considering the effects of TPA and FCA, the optical field propagation equation can be expressed in the following form

$$
\begin{aligned}
\frac{\partial A(z, \tau)}{\partial z}= & \left\{\frac{1}{2} \Gamma g_{0}(z, \tau)-\left.\Gamma \frac{i}{2} \frac{\partial g(\tau, \omega)}{\partial \omega}\right|_{\omega_{0}} \frac{\partial}{\partial \tau}\right. \\
& -\left.\frac{1}{4} \Gamma \frac{\partial^{2} g(\tau, \omega)}{\partial^{2} \omega}\right|_{\omega_{0}} \frac{\partial^{2}}{\partial^{2} \tau} \\
& -\frac{1}{2} \Gamma_{2} \beta_{2}\left(1+i \alpha_{2}\right)|A(z, \tau)|^{2} \\
& -\frac{1}{2} \Gamma \beta_{\mathrm{c}} n_{\mathrm{c}}(z, \tau)-\frac{1}{2} \Gamma \beta_{\mathrm{v}} n_{\mathrm{v}}(z, \tau) \\
& +\frac{1}{2} \beta_{\mathrm{GVD}} \frac{\partial^{2}}{\partial^{2} \tau}-\frac{1}{2} \alpha_{\mathrm{int}} \\
& +i \Phi(z, \tau)\}\} A(z, \tau),
\end{aligned}
$$

where $\Gamma$ is the mode confinement factor, $\Gamma_{2}$ the confinement factor for TPA, $\beta_{2}$ the TPA coefficient, $\beta_{\mathrm{GVD}}$ the group velocity dispersion coefficient, $\alpha_{\text {int }}$ the internal loss coefficient, $\alpha_{2}$ the linewidth enhancement factors related to TPA.

\section{Simulation results and analysis}

In simulations, the SOA is divided into 60 small sections and the values of physical parameters in each section are considered to be constant. The length of the active region is $300 \mu \mathrm{m}$, the volume of the active region is $60 \mu \mathrm{m}^{3}$, and the driven current to the SOA is $210 \mathrm{~mA}$. Values of other 
Table 1 Parameter values used in simulations

\begin{tabular}{|c|c|c|}
\hline Parameter & Symbol & Value \\
\hline Total density of states & $N_{0}$ & $3.244 \times 10^{5} \mu \mathrm{m}^{-3}$ \\
\hline $\begin{array}{l}\text { Group velocity dispersion coeffi- } \\
\text { cient }\end{array}$ & $\beta_{\mathrm{GVD}}$ & $5 \times 10^{6} \mathrm{ps}^{2} / \mathrm{mm}$ \\
\hline Carrier lifetime & $\tau_{\mathrm{s}}$ & $100 \mathrm{ps}$ \\
\hline $\begin{array}{l}\text { Carrier-carrier scattering time in } \\
\text { conduction band }\end{array}$ & $\tau_{\mathrm{c}}$ & $0.1 \mathrm{ps}$ \\
\hline $\begin{array}{l}\text { Carrier-carrier scattering time in } \\
\text { valence band }\end{array}$ & $\tau_{\mathrm{v}}$ & $0.05 \mathrm{ps}$ \\
\hline $\begin{array}{l}\text { Carrier-photon relaxation time in } \\
\text { conduction band }\end{array}$ & $\tau_{\mathrm{CR}}$ & $0.75 \mathrm{ps}$ \\
\hline $\begin{array}{l}\text { Carrier-photon relaxation time in } \\
\text { valence band }\end{array}$ & $\tau_{\mathrm{VR}}$ & $0.25 \mathrm{ps}$ \\
\hline Free-carrier absorption coefficient & $\beta_{\mathrm{c}}, \beta_{\mathrm{v}}$ & $1 \times 10^{-9} \mu \mathrm{m}^{-1}, 0 \mu \mathrm{m}^{-1}$ \\
\hline Two-photon absorption coefficient & $\beta_{2}$ & $3.6 \times 10^{-7} \mu \mathrm{m}^{2}$ \\
\hline Internal loss coefficient & $\alpha_{\text {int }}$ & $1.75 \times 10^{-3} \mu \mathrm{m}^{-1}$ \\
\hline Confinement factor & $\Gamma, \Gamma_{2}$, & $0.28,0.5$ \\
\hline Group velocity & $v_{\mathrm{g}}$ & $100 \mu \mathrm{m} / \mathrm{ps}$ \\
\hline Linewidth enhancement factor & $\alpha_{\mathrm{N}}$ & 7 \\
\hline Linewidth enhancement factor & $\alpha_{\mathrm{CH}}$ & 3 \\
\hline Linewidth enhancement factor & $\alpha_{\mathrm{SHB}}$ & 0.1 \\
\hline
\end{tabular}

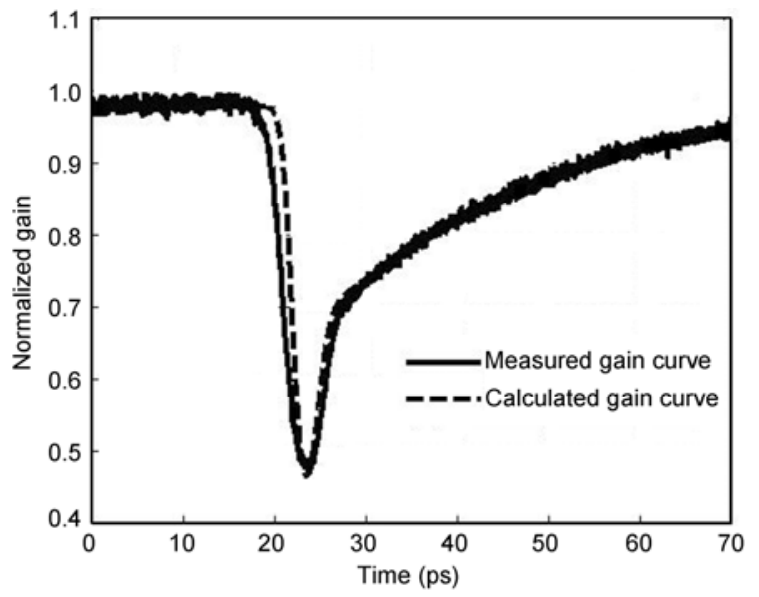

Figure 1 Normalized SOA gains induced by 2 ps pump pulses.

parameters are listed in Table 1; these have been used in a previous paper $[9,13,16]$. The equation can be solved using the finite-difference beam propagation method (FD-BMP).

The normalized gains induced by 2 ps pump pulses are shown Figure 1. The solid line represents the experimental result; the dash line gives the simulation result. Both results show reasonable accordance. The numerical model accurately characterizes the intra-band and inter-band carrier dynamics.

Figure 2 depicts the phase response of SOA at different pulse widths with a peak power of $18.1 \mathrm{~mW}$. In the figure, the phase dynamics have different features under different pump pulse width. Phase recovery, induced by the 3 ps pump pulses, is governed by a slow-recovery process consistent with the experimental result in [11]. With decreasing

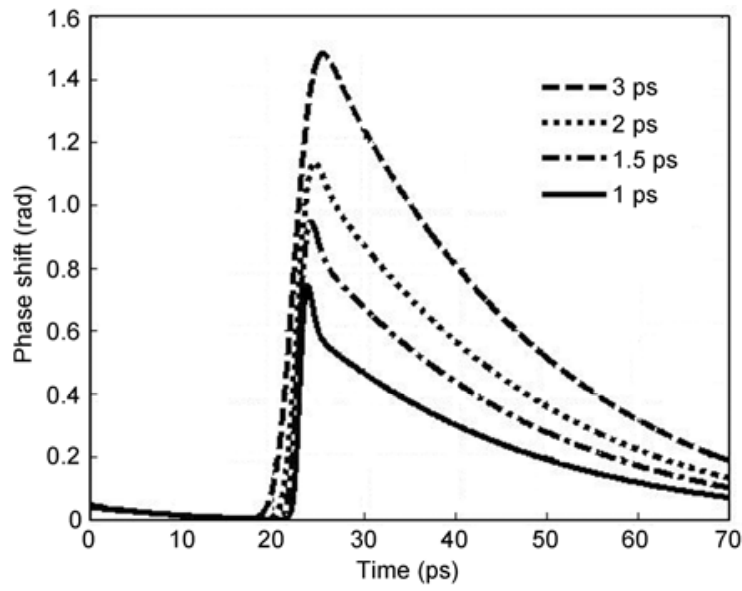

Figure 2 Phase shifts at different pump pulse widths with the same peak power.

pump pulse width, fast recovery appears gradually. The phase response induced by the 1 ps pump pulse width shows an obvious faster recovery. A similar experimental result was reported in [12]. The differences in phase recovery show the phase response of SOA is also related to operating conditions.

Carrier temperature dynamics of a SOA under different pump pulse widths with the same peak power of $18.1 \mathrm{~mW}$ are shown in Figure 3. The peak temperature of the conduction band and valence band is 322 and $300.7 \mathrm{~K}$ respectively. The carrier heating is mainly generated by conduction band carriers because the effective mass of electrons is smaller than of holes. Owing to this increase in carrier temperature, thermal equilibrium is achieved in the conductor band and valence band respectively; thus, in the process, the carrier temperature in each band rises above the lattice temperature, the carrier density decreases at the bottom of conduction band. Subsequently, carrier temperatures will gradually relax to the lattice temperature from interactions between carriers and phonons. The duration of carrier cooling is about 1 ps, resulting in an ultra-fast gain recovery. The impact of the $\mathrm{CH}$ on phase dynamics is smaller than carrier density changes because this process involves only a carrier transition from low level to high level, so a small $\alpha_{\mathrm{CH}}$ is adopted in simulation. For this reason, we see in Figure 2 a variation in the ultra-fast phase recovery compared with gain recovery at different pump pulse widths. Also in Figure 3, the carrier temperature curves only match the pulse shape, and there are no notable differences resulting from the underlying carrier temperature dynamics at different pulse widths. Therefore, $\mathrm{CH}$ effects are not a unique determining factor in ultra-fast phase recovery.

Figure 4 shows the contributions from the carrier density change, $\mathrm{CH}$, and $\mathrm{SHB}$ to gain suppression and the time scale for each physical effect. With the pump pulses injecting, $\mathrm{CH}$ and SHB mainly contribute to the gain suppression within the first few picoseconds, leading to an ultra-fast 

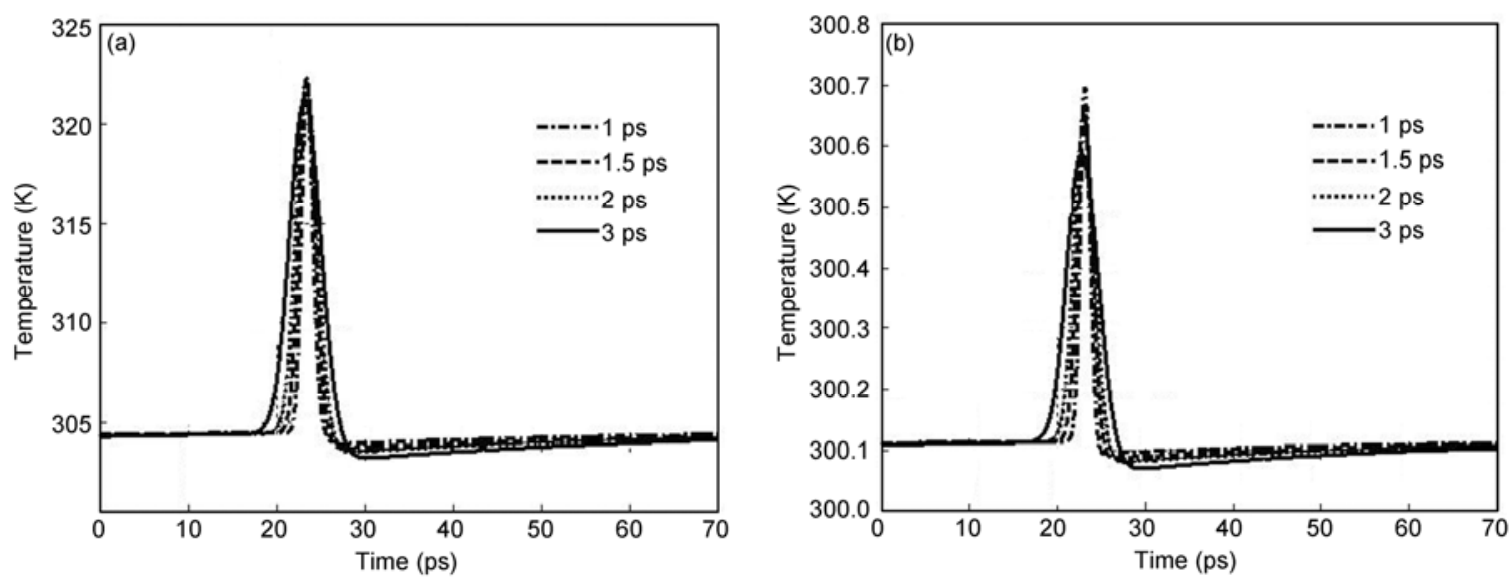

Figure 3 Carrier temperature at different pump pulse widths with the same peak power. (a) Carrier temperature in conduction band and (b) carrier temperature in valence band.

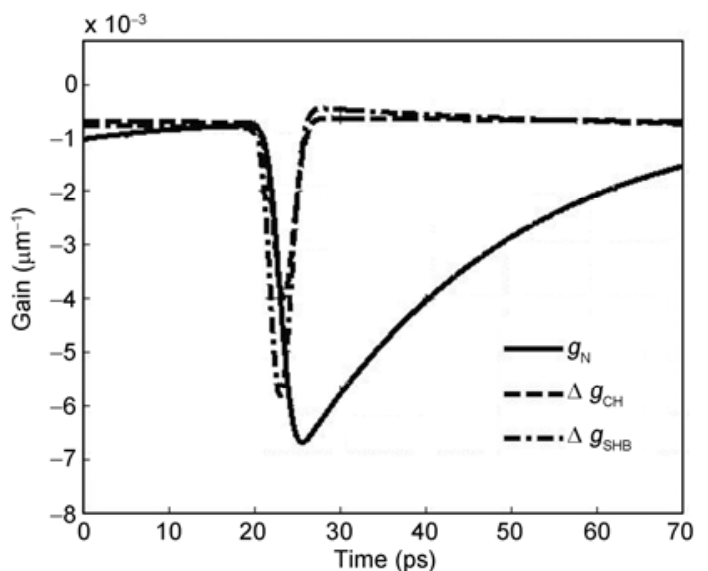

Figure 4 Normalized gains induced by carrier density change, $\mathrm{CH}$, and SHB

gain recovery process due to carrier-carrier and carrier-phonon scattering. From Figure 4, a delay exists between minimums of $g_{\mathrm{N}}$ and $\Delta g_{\mathrm{CH}}$. The delay is an important influence factor on ultra-fast phase recovery. Delaying on fast phase recovery can be explained as follows: If wide pump pulses are injected into an SOA, the delay is large enough with the carrier density change counteracting some of the impact of $\mathrm{CH}$ and SHB on phase shift. Consequently, fast phase recovery process disappears. If the pump pulse width is decreased, the reduced delay indicates that the inter-band and intra-band effects change in phase; thus a marked fast phase recovery appears with short pump pulses.

The SOA gain dynamics and phase shift at 2 ps pump pulses are shown in Figure 5(a). A delay exists between the maximum in the phase shift and the minimum of the gain; the results agree with those in $[17,18]$. The reason is that the phase shift and the gain dynamics are governed by inter-band effects and intra-band effects, respectively. Likewise, the delay between intra-band effects and inter-band effects results in a delay between gain and phase shift of SOA. In Figure 5(b), the delay change increases with pump pulse width.
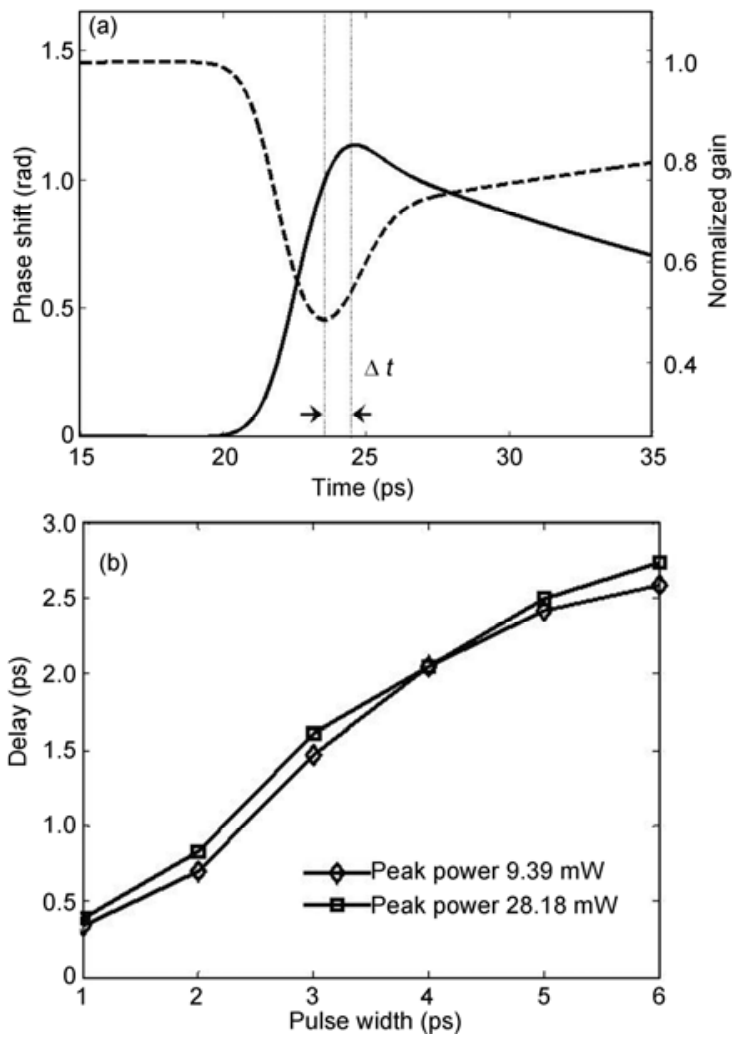

Figure 5 (a) Delay between phase dynamics (dash line) and gain dynamics (solid line); (b) delay variation with the pump pulse width for different peak pump pulse power.

\section{Conclusion}

We investigated the impact of the carrier depletion, carrier heating, and spectral hole burning on the phase dynamics of SOA. The results show that carrier heating and carrier depletion dominate at the different time scales during the recovery of carrier density, closely associating ultra-fast phase recovery with pump pulse width. If the pulse width is decreased, the delay is reduced, causing an ultra-fast phase 
recovery. Because the contributions of intra-band effects to phase shift is smaller than the gain, a delay also appears between phase dynamics and gain dynamics. Results from theoretical analysis can be used to analyze the performance of ultra-fast rate SOA-based optical signal processing devices, such as optical switches, optical logic gates, and optical add/drop multiplexers.

This work was supported by the National Basic Research Program of China (2011CB301705) and National Natural Science Foundation of China (60925019, 61090393 and 60907008).

1 Liu Y, Tangdiongga E, Li Z, et al. Error-free $320 \mathrm{~Gb} / \mathrm{s}$ all-optical wavelength conversion using a single semiconductor optical amplifier. J Lightwave Technol, 2007, 25: 103-108

2 Ni Y L, Wang Z Y. Experimental analysis of all optical wavelength conversion using XGM in SOA (in Chinese). Chin Sci Bull (Chin ver), 2009, 54: 3024-3029

3 Zhang X, Wang Y, Sun J, et al. All-optical AND gate at $10 \mathrm{Gbit} / \mathrm{s}$ based on cascaded single-port-couple SOAs. Opt Express, 2004, 12: 361-366

4 Dong J, Zhang X, Fu S, et al. Ultra fast all-optical signal processing based on single semiconductor optical amplifier and optical filtering. IEEE J Sel Top Quant, 2008, 14: 770-778

5 Duan P X, Chen L G, Zhang S J, et al. All-optical 2R regeneration based on self-induced polarization rotation in a single semiconductor optical amplifier. Chin Sci Bull, 2009, 54: 3704-3708

6 Tangdiongga E, Liu Y, De W H, et al. All-optical demultiplexing of 640-to-40-Gb/s using filtered chirp of a semiconductor optical amplifier. Opt Lett, 2007, 32: 835-837

7 Nicholes S C, Masanovic M L, Jevremovic B, et al. An 8x8 InP monolithic tunable optical router (MOTOR) packet forwarding chip. J Lightwave Technol, 2010, 28: 641-650

8 Huang X, Qin C, Huang D, et al. Local carrier recovery acceleration in quantum well semiconductor optical amplifiers. IEEE J Quantum Electron, 2010, 46: 1407-1413

9 Nielsen M L, Mørk J. Experimental and theoretical investigation of the impact of ultra-fast carrier dynamics on high speed SOA-based all-optical switches. Opt Express, 2006, 14: 331-347

10 Schares L, Schubert C, Schmidt C, et al. Phase dynamics of semiconductor optical amplifiers at 10-40 GHz. IEEE J Quantum Electron, 2003, 39: 1394-1408

11 Giller R, Manning R J, Cotter D. Gain and phase recovery of optically excited semiconductor optical amplifiers. IEEE Photonic Technol Lett, 2006, 18: 1061-1063

12 Gomez-Iglesias A, Fenn J G, Mazilu M, et al. Carrier heating in semiconductor optical amplifier-based sagnac-type all-optical switches. Semicond Sci Tech, 2006, 21: 1703-1708

13 Hong M Y, Chang Y H, Dienes A, et al. Femtosecond self- and cross-phase modulation in semiconductor laser amplifiers. IEEE J Sel Top Quant, 1996, 2: 523-539

14 Agrawal G P. Effect of gain dispersion on ultrashort pulse amplification in semiconductor laser amplifiers. IEEE J Quantum Electron, 1991, 2: 1843-1849

15 Dienes A, Heritage J P, Hong M Y, et al. Time- and spectral-domain evolution of sub-picosecond pulses in semiconductor optical amplifiers. Opt Lett, 1992, 17: 1602-1604

16 Mark J, Mørk J. Subpicosecond gain dynamics in InGaAsP optical amplifiers: Experiment and theory. Appl Phys Lett, 1992, 61: 22812283

17 Wang J, Maitra A, Poulton C G, et al. Temporal dynamics of the alpha factor in semiconductor optical amplifiers. J Lightwave Technol, 2007, 25: 891-900

18 Dailey J M, Koch T L. Impact of carrier heating on SOA dynamics for wavelength conversion. In: Proceeding of lasers and electrooptical society, Montreal, Qua, IEEE, 2006 Oct. 156-157

Open Access This article is distributed under the terms of the Creative Commons Attribution License which permits any use, distribution, and reproduction in any medium, provided the original author(s) and source are credited. 